\title{
An Investigation of Critical Failure Factors In Information Technology Projects
}

\author{
Dr. Roslina Ibrahim ${ }^{1}$,Erfan Ayazi ${ }^{1}$,Shahab Nasrmalek ${ }^{1}$,Shamin Nakhat ${ }^{2}$ \\ ${ }^{1}$ Advanced Informatics School (AIS) - Universiti Teknologi Malaysia (UTM), Kuala Lumpur, Malaysia \\ ${ }^{2}$ Razak School of Engineering and Advanced Technology(UTM Razak)- Universiti Teknologi Malaysia \\ (UTM)Kuala Lumpur, Malaysia
}

\begin{abstract}
Rate of failed projects in information technology system project remains high in comparison with other infrastructure or high technology projects. The objective of this paper is to determine and represent a broad range of potential failure factors during the implementation phase and cause of IS/IT Project defeat/failure. Challenges exist in order to achieve the projects goal successfully and to avoid the failure. In this research study, 12 articles were studied as significant contributions to analyze developing a list of critical failure factors of IT projects
\end{abstract}

Keywords: Project failure, IS/IT projects,

\section{Introduction}

Computerized information systems are pervasive in all forms of business organizations. Recent studies show that many of these projects have 'failed', in the combination of budget and/or schedule overruns and/or for not meeting users' requirements [8]. The well known and now widely quoted Chaos Report by Standish Group [17] declared that IT projects are in chaos. Table 1 provides a summarized report card on project outcomes based on the Report.

\begin{tabular}{|l|l|l|l|l|l|l|l|}
\hline Benchmark/year & 1994 & 1996 & 1998 & 2000 & 2004 & 2006 & 2008 \\
\hline Succeeded (\%) & 16 & 27 & 26 & 28 & 29 & 35 & 32 \\
\hline Challenged (\%) & 53 & 33 & 46 & 49 & 53 & 46 & 44 \\
\hline Failed (\%) & 31 & 40 & 28 & 23 & 18 & 19 & 24 \\
\hline
\end{tabular}

Table1: Standish IT project performance over a decade [17]

\section{Objectives and Methodology}

The objectives of the study are: To identify the relative important failure attributes; and to understand the latent properties of these failure attributes by studying the critical failure factors for further suggestions to improve the performance. For the study, a huge amount of documented data on completed projects is required (studied). This study makes use of a literature review research method. In particular, it examines and discusses thirteen critical failure factors contributing to failed implementation.

\section{Literture Reviw And Data Collection}

IT projects are unique in their nature and management. PMBOK 2003 defines project management as "the application of knowledge, skills, tools, and techniques to project activities to meet project requirements". The project requirements or objectives vary from project to project and person to person. The attributes _also referred to as "factor" in some literature_ responsible for achievement of these requirements and the attributes obstructing the achievement of these requirements have fascinated the researchers since the 1960s. Rockart 1982[18] first used critical success factor (CSF) defined it in the context of information systems and project management.

Rowlinson [19] in 1999 states that "Critical success factors are those fundamental issues inherent in the project, which must be maintained in order for team working to take place in an efficient and effective manner. They require day-to-day attention and operate throughout the life of the project".

Chan et al. 2001 [15] investigated the project success factors for design and build_D\&B_ projects and identified six project success factors. These are project team commitment, client's competencies, contractor's competencies, risk and liability assessment, Endusers' needs, and constraints imposed by end-users. Further they found project team commitment, client's competencies, and contractor's competencies to be important to bring a successful project outcome. 


\section{Concept of Information Systems and Technology}

In the modern economy of today's world, enterprises are largely investing in information systems and technology and particularly in the ways these can help them managing their businesses. This transition has appeared to be an indispensable change in most of prosperous companies on the ground that it is increasingly believed these investments could be rich sources of competitive advantage [20].

The term 'Information Systems' has originally born to refer to any wide variety of computing hardware, communication technology and software combinations designed to manipulate information related to certain business processes [21].

It serves to coordinate the work of many different organizational functions, from a back office administration support, to a company's strategic management tool. The payroll, sales orders, inventory control and personnel records systems are some examples of back office administration support systems. An information system stores, processes and delivers information relevant to an organization, in such a way that the information is useful to those who wish to use it, including managers, staff, customers, and suppliers. An information system may or may not involve the use of computer systems [4].

\section{Notions of IS/IT failure}

Lysine and Hir Schheim [16] defined four major notions or categories of IS failures as follows:

1. Correspondence Failure: When the systems design objectives are not met, the information system is considered a failure.

2. Process Failure: A process failure occurs when an IS cannot be developed within an allocated budget, and/or time schedule.

3. Interaction Failure: The level of end-user usage of the information system is suggested as a surrogate in IS performance measurement. Heavy usage does not necessarily mean high user satisfaction and improved task performance.

4. Expectation Failure: The notion of expectation failure views failure as the inability of a system to meet its stakeholders' requirements, expectations, or values.

Flowers [21] defined an information system as a failure if any of these following situations occurs:

Firstly when the system as a whole does not operate as expected and its overall performance is sub-optimal.

Secondly, if on implementation, it does not perform as originally intended or if it is so user-hostile that it is rejected by users and underutilized.

Thirdly if, the cost of the development exceeds any benefits the system may bring throughout its useful life.

Finally due to problems with the complexity of the system, or the management of the project, the information system development is abandoned before it is completed.

\section{Failure factors in researches}

Failure or Problem research is typically based on "lessons learned" from certain types of projects, but they are mostly similar enough to be generalized. Reel (1999) [22] focuses more on generic software development projects and compiles 10 signs of software development project failure, at least seven of which are determined even before a design is developed or a single line of code is written. Tsun Chow and Dac-Buu Cao(2007) [2] study problems in transforming organizations to agile processes, while Yongyi Shod, Ying Ying, (2005)[5] discusses in detail mistakes and misunderstandings occurred in agile projects. A research by Winters, F. (2003)[9] emphasizes on management challenges in implementing agile projects, whereas a study by Marchewka (2006)[14] covers problems not only in management aspect but also in people, process, and technology dimensions of migrating to agile projects.

\begin{tabular}{|c|c|c|c|c|c|}
\hline $\begin{array}{c}\text { K. C. Iyer1 and } \\
\text { K. N. Jha2, 2006 }\end{array}$ & $\begin{array}{c}\text { Tsun Chow, Dac- } \\
\text { Buu Cao, 2007 }\end{array}$ & K.T. Yeo.2002 & $\begin{array}{c}\text { Wong, A., } \\
\text { Scarbrough } \\
\mathbf{2 0 0 5}\end{array}$ & $\begin{array}{c}\text { Yongyi Shod, } \\
\text { Ying Ying2, 2005 }\end{array}$ & $\begin{array}{c}\text { Kaur, B. P., } \\
\text { Aggarwal, H., \& } \\
\text { Singh, G 2004 }\end{array}$ \\
\hline $\begin{array}{c}\text { Conflict Among } \\
\text { Project } \\
\text { Participants }\end{array}$ & $\begin{array}{c}\text { Lack of executive } \\
\text { sponsorship }\end{array}$ & $\begin{array}{c}\text { Underestimate of } \\
\text { timeline }\end{array}$ & $\begin{array}{c}\text { ERP system } \\
\text { misfit }\end{array}$ & $\begin{array}{c}\text { Weak definition } \\
\text { of requirements } \\
\text { and scope }\end{array}$ & $\begin{array}{c}\text { Poorly stated } \\
\text { project goals }\end{array}$ \\
\hline $\begin{array}{c}\text { Project Manager's } \\
\text { Ignorance }\end{array}$ & $\begin{array}{c}\text { Lack of } \\
\text { management } \\
\text { commitment }\end{array}$ & $\begin{array}{c}\text { Weak definitions } \\
\text { of requirements } \\
\text { and scopes }\end{array}$ & $\begin{array}{c}\text { High turnover } \\
\text { rate of project } \\
\text { team members }\end{array}$ & $\begin{array}{c}\text { Reactive and not } \\
\text { proactive in } \\
\text { problem solving }\end{array}$ & $\begin{array}{c}\text { Poor project team } \\
\text { composition }\end{array}$ \\
\hline $\begin{array}{c}\text { Hostile } \\
\text { Environment }\end{array}$ & $\begin{array}{c}\text { Organizational } \\
\text { culture too } \\
\text { traditional }\end{array}$ & $\begin{array}{c}\text { Inadequate } \\
\text { project risk } \\
\text { analysis }\end{array}$ & $\begin{array}{c}\text { Poor consultant } \\
\text { effectiveness }\end{array}$ & $\begin{array}{c}\text { Poor or lack of } \\
\text { business process } \\
\text { reengineering }\end{array}$ & $\begin{array}{c}\text { Lack of project } \\
\text { management and } \\
\text { control }\end{array}$ \\
\hline $\begin{array}{c}\text { Owner's } \\
\text { Incompetence }\end{array}$ & $\begin{array}{c}\text { Organizational } \\
\text { culture too political }\end{array}$ & $\begin{array}{c}\text { assumptions } \\
\text { regarding risk } \\
\text { analysis }\end{array}$ & $\begin{array}{c}\text { Pnderestimate the } \\
\text { gap between } \\
\text { technology } \\
\text { ability }\end{array}$ & $\begin{array}{c}\text { Little technical } \\
\text { know-how }\end{array}$ \\
\hline
\end{tabular}




\begin{tabular}{|c|c|c|c|c|c|}
\hline $\begin{array}{c}\text { Indecisiveness of } \\
\text { Project } \\
\text { Participants }\end{array}$ & $\begin{array}{c}\text { Organizational size } \\
\text { too large }\end{array}$ & $\begin{array}{l}\text { Ambiguous } \\
\text { business needs } \\
\text { and unclear } \\
\text { vision }\end{array}$ & $\begin{array}{l}\text { Poor knowledge } \\
\text { transfer }\end{array}$ & $\begin{array}{l}\text { Unrealistic } \\
\text { expectation of the } \\
\text { information } \\
\text { system }\end{array}$ & $\begin{array}{c}\text { Poor technology } \\
\text { base or } \\
\text { infrastructure }\end{array}$ \\
\hline $\begin{array}{l}\text { Harsh Climatic } \\
\text { Condition at Site }\end{array}$ & $\begin{array}{l}\text { Lack of agile } \\
\text { logistical } \\
\text { arrangements }\end{array}$ & $\begin{array}{c}\text { Lack user } \\
\text { involvement and } \\
\text { inputs from the } \\
\text { onset }\end{array}$ & $\begin{array}{l}\text { Poor project } \\
\text { management } \\
\text { effectiveness }\end{array}$ & $\begin{array}{c}\text { Ineffective } \\
\text { internal } \\
\text { communication }\end{array}$ & $\begin{array}{l}\text { Lack of senior } \\
\text { management } \\
\text { involvement }\end{array}$ \\
\hline $\begin{array}{c}\text { Project Specific } \\
\text { Factor }\end{array}$ & $\begin{array}{c}\text { Lack of necessary } \\
\text { skill-set }\end{array}$ & $\begin{array}{c}\text { Top down } \\
\text { management } \\
\text { style }\end{array}$ & $\begin{array}{c}\text { Poor quality of } \\
\text { Business Process } \\
\text { Reengineering }\end{array}$ & $\begin{array}{l}\text { Involvement of } \\
\text { high degree } \\
\text { customization }\end{array}$ & $\begin{array}{c}\text { Escalating project } \\
\text { cost and time of } \\
\text { completion }\end{array}$ \\
\hline $\begin{array}{l}\text { Project manager's } \\
\text { ignorance and } \\
\text { lack of knowledge }\end{array}$ & $\begin{array}{c}\text { Lack of project } \\
\text { management } \\
\text { competence }\end{array}$ & $\begin{array}{l}\text { Poor internal } \\
\text { communication }\end{array}$ & $\begin{array}{l}\text { Poor quality of } \\
\text { testing }\end{array}$ & $\begin{array}{l}\text { Organizational } \\
\text { rigidity and } \\
\text { bureaucracy }\end{array}$ & \\
\hline \multirow[t]{3}{*}{$\begin{array}{c}\text { Aggressive } \\
\text { competition } \\
\text { during tender } \\
\text { stage } \\
\end{array}$} & Lack of team work & $\begin{array}{l}\text { Absence of an } \\
\text { influential } \\
\text { champion and } \\
\text { change agent }\end{array}$ & $\begin{array}{c}\text { Poor top } \\
\text { management } \\
\text { support }\end{array}$ & $\begin{array}{c}\text { Insufficient } \\
\text { authority of the } \\
\text { project manager }\end{array}$ & \\
\hline & $\begin{array}{c}\text { Resistance from } \\
\text { groups or } \\
\text { individuals }\end{array}$ & $\begin{array}{l}\text { Reactive and not } \\
\text { pro-active in } \\
\text { dealing with } \\
\text { problems }\end{array}$ & $\begin{array}{l}\text { Too tight project } \\
\text { schedule }\end{array}$ & $\begin{array}{l}\text { Lack of support } \\
\text { from middle-level } \\
\text { function managers }\end{array}$ & \\
\hline & $\begin{array}{l}\text { Bad customer } \\
\text { relationship }\end{array}$ & $\begin{array}{l}\text { Consultant/vendo } \\
\text { r underestimated } \\
\text { the project scope } \\
\text { and complexity }\end{array}$ & $\begin{array}{l}\text { Unclear concept } \\
\text { of the nature and } \\
\text { use of ERP } \\
\text { system from the } \\
\text { users' perspective }\end{array}$ & & \\
\hline $\begin{array}{l}\text { Alimohammadin } \\
\text { avid, R. (2007). }\end{array}$ & $\begin{array}{l}\text { Nasir, M. H. N., } \\
\text { \& Sahibuddin, S. } \\
\text { (2011). }\end{array}$ & $\begin{array}{l}\text { Winters, F. } \\
(2003) .\end{array}$ & $\begin{array}{c}\text { Marchewka, J. } \\
\text { T. (2006 }\end{array}$ & Garg, P. (2010) & E.J. Umble 2003 \\
\hline $\begin{array}{c}\text { Lack of } \\
\text { commitment from } \\
\text { organizational top } \\
\text { management to } \\
\text { support IS/IT } \\
\text { projects }\end{array}$ & $\begin{array}{l}\text { Support from top } \\
\text { management }\end{array}$ & $\begin{array}{l}\text { Lack of User } \\
\text { Involvement }\end{array}$ & $\begin{array}{l}\text { Incomplete } \\
\text { requirements }\end{array}$ & $\begin{array}{l}\text { Lack of top } \\
\text { management } \\
\text { commitment }\end{array}$ & unclear Goals \\
\hline $\begin{array}{l}\text { General senior } \\
\text { management's } \\
\text { lack of knowledge } \\
\text { about structures } \\
\text { and functions } \\
\text { ofIS/IT }\end{array}$ & $\begin{array}{l}\text { User/client } \\
\text { involvement }\end{array}$ & $\begin{array}{l}\text { Long or } \\
\text { Unrealistic Time } \\
\text { Scales }\end{array}$ & $\begin{array}{l}\text { Lack of user } \\
\text { involvement }\end{array}$ & $\begin{array}{l}\text { Poor middle } \\
\text { management } \\
\text { commitment }\end{array}$ & $\begin{array}{l}\text { top management } \\
\text { commitment }\end{array}$ \\
\hline $\begin{array}{c}\text { Conflicting } \\
\text { decentralized } \\
\text { decision-making } \\
\text { systems in } \\
\text { organizations for } \\
\text { IS/IT projects }\end{array}$ & $\begin{array}{l}\text { Committed and } \\
\text { motivated team }\end{array}$ & $\begin{array}{c}\text { Poor or No } \\
\text { Requirements }\end{array}$ & $\begin{array}{l}\text { Lack of } \\
\text { resources }\end{array}$ & $\begin{array}{l}\text { inadequate } \\
\text { functional } \\
\text { requirements }\end{array}$ & $\begin{array}{l}\text { poor Project } \\
\text { Manager }\end{array}$ \\
\hline $\begin{array}{l}\text { Cultural issues in } \\
\text { acceptance and } \\
\text { making proper } \\
\text { use of IS/IT } \\
\text { systems in } \\
\text { organizations } \\
\end{array}$ & $\begin{array}{l}\text { Unclear } \\
\text { requirements and } \\
\text { specifications }\end{array}$ & Scope Creep & $\begin{array}{c}\text { Unrealistic } \\
\text { expectations }\end{array}$ & $\begin{array}{c}\text { Over-reliance on } \\
\text { heavy } \\
\text { customization }\end{array}$ & $\begin{array}{l}\text { Organizational } \\
\text { resistance }\end{array}$ \\
\hline $\begin{array}{l}\text { Lack of expertise } \\
\text { in terms of project } \\
\text { management and } \\
\text { IS/IT knowledge } \\
\text { \& techniques }\end{array}$ & $\begin{array}{l}\text { Unrealistic } \\
\text { schedule }\end{array}$ & $\begin{array}{c}\text { No Change } \\
\text { Control System }\end{array}$ & $\begin{array}{c}\text { Lack of } \\
\text { executive support }\end{array}$ & Inaccurate data & $\begin{array}{l}\text { inadequate } \\
\text { training }\end{array}$ \\
\hline $\begin{array}{l}\text { Conflicting goals } \\
\text { and } \\
\text { miscommunicatio } \\
\mathrm{n} \text { between } \\
\text { department } \\
\text { managers and } \\
\text { project managers }\end{array}$ & $\begin{array}{l}\text { Unfrozen } \\
\text { requirement }\end{array}$ & Poor Testing & $\begin{array}{l}\text { Changing } \\
\text { requirements \& } \\
\text { specifications }\end{array}$ & $\begin{array}{l}\text { Poor quality of } \\
\text { testing }\end{array}$ & poor team \\
\hline $\begin{array}{l}\text { Overlaps of } \\
\text { planning, design, } \\
\text { implementation, } \\
\text { controlling and } \\
\text { operation phases } \\
\text { in IS/IT projects }\end{array}$ & $\begin{array}{l}\text { Inadequate } \\
\text { resources }\end{array}$ & & Lack of planning & $\begin{array}{l}\text { Poor consultant } \\
\text { effectiveness }\end{array}$ & wrong Data \\
\hline
\end{tabular}




\begin{tabular}{|c|c|c|c|c|}
\hline $\begin{array}{l}\text { Dysfunctional } \\
\text { implementation \& } \\
\text { operation phases } \\
\text { in IS/IT projects }\end{array}$ & $\begin{array}{l}\text { Poor quality } \\
\text { management }\end{array}$ & $\begin{array}{l}\text { Didn't need it } \\
\text { any longer }\end{array}$ & $\begin{array}{c}\text { Poor IT } \\
\text { infrastructure }\end{array}$ & $\begin{array}{l}\text { technical } \\
\text { difficulties }\end{array}$ \\
\hline $\begin{array}{c}\text { Long term } \\
\text { investment to } \\
\text { reach economic } \\
\text { efficiency }\end{array}$ & $\begin{array}{l}\text { End-user training } \\
\text { provision }\end{array}$ & $\begin{array}{l}\text { Lack of IT } \\
\text { management }\end{array}$ & $\begin{array}{c}\text { Users' resistance } \\
\text { to change }\end{array}$ & \\
\hline \multirow[t]{2}{*}{$\begin{array}{l}\text { Lack of expertise } \\
\text { in terms of project } \\
\text { management and } \\
\text { IS/IT experience }\end{array}$} & $\begin{array}{l}\text { Supporting tools } \\
\text { and poor } \\
\text { infrastructure }\end{array}$ & $\begin{array}{l}\text { Technology } \\
\text { illiteracy }\end{array}$ & $\begin{array}{l}\text { High Attrition } \\
\text { rate of project } \\
\text { team members }\end{array}$ & \\
\hline & & & $\begin{array}{l}\text { Inadequate } \\
\text { resources }\end{array}$ & \\
\hline
\end{tabular}

Table2: Failure Factors and The authors

Based on the literature mentioned in Table 2, we classified failures/problems into five categories: Factors related to the project, project manager, team member, organization and environment. The related factors for each are written below:

Factors related to project: Size \& value, Uniqueness of project activities, Density of a project, Life cycle and Urgency.

Factors related to the project manager: Ability to delegate authority, Ability to trade off, Ability to coordinate, Perception of his role and responsibilities and Competence Commitment.

Project team members: Technical background, Communication, Trouble shooting and Commitment

Factors related to the organization: Top management support, Project organizational structure, Functional managers' support and Project champion.

Factors related to the environment: Political environment, Economic environment, Social environment, Technological environment, Nature, Client, Competitors and Subcontractors.

Findings

Based on the research study shown in table 3 , there are four common factors that can be summarized as Poor top management support, Poor consultant effectiveness, poor project management effectiveness and Lack of User Involvement; each of these factors are described as follows:

Poor top management support

Top management is expected to provide support in the areas of committing to any IT project, sufficient financial and human resource, and the resolution of political problems if necessary. As an Example: limited financial support contributed to a rushed ERP implementation process project team members were overloaded and thus high staff turnover rate, ineffective knowledge transfer, and political problems occurred. Insufficient commitment could lead to political problems which hindered the implementation process [3][4].

\section{Poor consultant effectiveness}

The results show consultants were considered by successful project team members to be inexperienced and unable to provide a professional level of advice IT project planning [3]. Consultants may communicated ineffectively during the project phase due to language barriers, and only suggested workarounds without applying professional skills to conduct IT projects.[3][4]

\section{Poor project management effectiveness}

The majority of researcher agreed that a failure to plan, lead, manage and monitor the project was a core factor that resulted in their implementation failure, because the IT project was complex, and This factor explain project manager's competence as key to success of the project. A competent manager has the technical capability and monitoring capabilities. He makes his people committed for the project through effective leadership and by acting in nonpartisan ways. He shows his trust in his project team by way of delegating the authority to his team. He organizes resources through constant persuasion with his higher ups, he takes active part in construction control meetings held at site level, and he acts as a catalyst in training his human resources in the skill demanded by the project. All these attributes can be thought of originating from project manager's competence, hence the name.project teams were required to collaborate with top management, different departments, users and consultants during implementation process. The ERP project was considered by the project managers to be challenging and demanding, as it involved managing systems, people (project team, users and external consultant) as well as re-designing business processes [1][3][5].

\section{Lack of User Involvement}

Lack of user involvement has proved fatal for many projects. Without user involvement nobody in the business feels committed to a system, and can even be hostile to it. If a project is going to be a success, senior 
management and users need to be involved from the start, and continuously throughout the development. This requires time and effort, and when the people in a business are already stretched, finding time for a new project is not high on their priorities. Therefore senior management need to continuously support the project to make it clear to staff it is a priority [9].

\begin{tabular}{|c|c|c|c|c|}
\hline \multirow[t]{2}{*}{ Rank } & \multirow[t]{2}{*}{ Critical Failure factors } & \multirow[t]{2}{*}{ Literature Citation } & \multicolumn{2}{|c|}{$\begin{array}{l}\text { Citation count in } \\
\text { the literature }(n= \\
12)\end{array}$} \\
\hline & & & Frequency & $\%$ \\
\hline 4 & project team members & $\begin{array}{l}\text { Tsun Chow, Dac-Buu Cao, 2007,K.T. Yeo.2002,Wong, } \\
\text { A., Scarbrough ,2005 , Nasir, M. H. N., \& Sahibuddin, S. } \\
\text { (2011),Winters, F. (2003),Marchewka, J. T. (2006 }\end{array}$ & 5 & 42 \\
\hline 2 & $\begin{array}{l}\text { Poor consultant } \\
\text { effectiveness }\end{array}$ & $\begin{array}{l}\text { Wong, A., Scarbrough ,2005,Yongyi Shod, Ying Ying2, } \\
\text { 2005,Kaur, B. P., Aggarwal, H., \& Singh, G } \\
\text { 2004,Alimohammadinavid, R. (2007). Nasir, M. H. N., } \\
\text { \& Sahibuddin, S. (2011).Winters, F. (2003),Marchewka, } \\
\text { J. T. (2006),Garg, P. (2010) }\end{array}$ & 8 & 67 \\
\hline 5 & Poor IT infrastructure & $\begin{array}{l}\text { K.T. Yeo.2002,Wong, A., Scarbrough ,2005, Nasir, M. H. } \\
\text { N., \& Sahibuddin, S. (2011). Garg, P. (2010) }\end{array}$ & 4 & 33 \\
\hline 6 & Poor knowledge transfer & $\begin{array}{c}\text { Wong, A., Scarbrough ,2005,Marchewka, J. T. } \\
\text { (2006),Garg, P. (2010) }\end{array}$ & 3 & 25 \\
\hline 2 & $\begin{array}{l}\text { Poor project } \\
\text { management } \\
\text { effectiveness }\end{array}$ & $\begin{array}{l}\text { K. C. Iyer1 and K. N. Jha2, 2006,Tsun Chow, Dac-Buu } \\
\text { Cao, 2007,Wong, A., Scarbrough ,2005, Kaur, B. P., } \\
\text { Aggarwal, H., \& Singh, G } 2004 \text {,Alimohammadinavid, } \\
\text { R. (2007). ,Marchewka, J. T. (2006),Garg, P. (2010),E.J. } \\
\text { Umble }\end{array}$ & 8 & 67 \\
\hline 7 & $\begin{array}{l}\text { Poor quality of Business } \\
\text { Process Reengineering }\end{array}$ & $\begin{array}{c}\text { Wong, A., Scarbrough ,2005,Yongyi Shod, Ying Ying2, } \\
2005\end{array}$ & 2 & 17 \\
\hline 1 & $\begin{array}{l}\text { Poor top management } \\
\text { support }\end{array}$ & $\begin{array}{l}\text { Tsun Chow, Dac-Buu Cao, 2007,K.T Yeo.2002,Wong, } \\
\text { A., Scarbrough ,2005 ,Yongyi Shod, Ying Ying2, } \\
\text { 2005,Kaur, B. P., Aggarwal, H., \& Singh, G } \\
\text { 2004,Alimohammadinavid, R. (2007). Nasir, M. H. N., } \\
\text { \& Sahibuddin, S. (2011).Marchewka, J. T. (2006),Garg, } \\
\text { P. (2010),E.J. Umble }\end{array}$ & 10 & 83 \\
\hline 6 & $\begin{array}{l}\text { Too tight project } \\
\text { schedule }\end{array}$ & $\begin{array}{c}\text { Tsun Chow, Dac-Buu Cao, 2007,K.T. Yeo.2002,Winters, } \\
\text { F. (2003). }\end{array}$ & 3 & 25 \\
\hline 5 & $\begin{array}{l}\text { Unclear concept of } \\
\text { Goals }\end{array}$ & $\begin{array}{c}\text { Yongyi Shod, Ying Ying2, 2005,Kaur, B. P., Aggarwal, } \\
\text { H., \& Singh, G 2004,K.T. Yeo.2002,Kaur, B. P., } \\
\text { Aggarwal, H., \& Singh, G } 2004\end{array}$ & 4 & 33 \\
\hline 7 & Unrealistic expectations & $\begin{array}{c}\text { Wong, A., Scarbrough ,2005, Yongyi Shod, Ying } \\
\text { Ying2, } 2005\end{array}$ & 2 & 17 \\
\hline 4 & $\begin{array}{l}\text { Users' resistance to } \\
\text { change }\end{array}$ & $\begin{array}{c}\text { Tsun Chow, Dac-Buu Cao, 2007,K.T. Yeo.2002,Wong, } \\
\text { A., Scarbrough ,2005 ,Nasir, M. H. N., \& Sahibuddin, S. } \\
\text { (2011).E.J. Umble }\end{array}$ & 5 & 42 \\
\hline
\end{tabular}




\begin{tabular}{|c|c|c|c|}
7 & $\begin{array}{c}\text { Poor internal } \\
\text { comminucation }\end{array}$ & K.T. Yeo.2002, Yongyi Shod, Ying Ying2, 2005 & 2 \\
\hline 3 & $\begin{array}{l}\text { Lack of user } \\
\text { Involvement }\end{array}$ & $\begin{array}{r}\text { Tsun Chow, Dac-Buu Cao, 2007,E.J. Umble , Garg, P. } \\
\text { (2010),Marchewka, J. T. (2006), Winters, F. } \\
\text { (2003),Nasir, M. H. N., \& Sahibuddin, S. (2011). }\end{array}$ & 6 \\
\hline
\end{tabular}

Table3: Common failure factors and their ranks

The factors those are presented in Table 3 are not the only ones that affect the success or failure of a project, but in many studies and reports they appear near, or at the top of the list. They are all interlinked, but as it can be seen they are not technical issues, but management and training ones. This supports the idea that IT projects should be treated as business projects.

\section{Conclusion}

This study makes use of a literature review research method and follows IT project life cycle to identify IT/IS associated problems. More importantly, it examines and discusses fourteen critical failure factors contributing to failed implementation. The results of this research suggest that the role performed by Top Management to Support (involvement and Participation) is important for avoiding the failure within the different IT project implementation. Project managers should exercise effective control and monitoring of the project and consultant effectiveness. There is a good opportunity, if an organization or project manager is attentive, to control the top four critical factors to drive towards project success All these critical factors which were cited the most, classified into four factors: Poor top management support, Poor consultant effectiveness, poor project management effectiveness and Lack of User Involvement.

\section{References}

[1] K.C. Iyer, K.N. Jha Critical Factors affecting schedule performance: evidence from Indian construction projects ASCE J Constr Eng Manage, 132 (8) (2006), pp. 871-881

[2] Chow, T., and Cao, D.-B. 2008. "A Survey Study of CriticalSuccess Factors in Agile Software Projects," The Journal of Systems and Software (81:6), pp. 961-971

[3] K. Yeo Critical failure factors in information systems projects Int J Project Manage, 20 (2000), pp. 241-246

[4] Wong, A. et al. (2005) "Critical Failure Factors in ERP Implementation", Pacific Asia Conference on Information Systems (PACIS)

[5] y. shou and y. ying, "Critical Failure Factors of Information System Projects in Chinese Enterprises," presented at International conference on service systems and services management, 2005.

[6] Kaur, B. P., Aggarwal, H., \& Singh, G. Information System Planning Failures in Indian Telecommunication Industry.

[7] Alimohammadinavid, R. (2007). IS/IT Projects Failure:-An Investigation in Iranian Organizations.

[8] Nasir, M. H. N., \& Sahibuddin, S. (2011). Critical success factors for software projects: A comparative study. Scientific Research and Essays, 6(10), 2174-2186.

[9] Winters, F. (2003). " The top ten reasons projects fail(part 7). Retrieved March, 10, 2005.

[10] Marchewka, J. T. (2006). Information technology project management. John Wiley \& Sons.

[11] Al Neimat, T. (2005). Why IT projects fail. The Project Perfect White Paper Collection. Retrieved November, 1, 2011.

[12] Garg, P. (2010). Critical Failure Factors for Enterprise Resource Planning Implementations in Indian Retail Organizations: An Exploratory Study. Journal of Information Technology Impact, 10(1), 35-44.

[13] Umble, E. J., Haft, R. R., \& Umble, M. M. (2003). Enterprise resource planning: Implementation procedures and critical success factors. European journal of operational research, 146(2), 241-257.

[14] Matthews, J., \& Rowlinson, S. (1999). Partnering: incorporating safety management. Engineering Construction and Architectural Management, 6(4), 347-357.

[15] Chan, A. P., Ho, D. C., \& Tam, C. M. (2001). Design and build project success factors: Multivariate analysis. Journal of construction engineering and management, 127(2), 93-100.

[16] Lyytinen, K., \& Hirschheim, R. (1987). Information systems failures: a survey and classification of the empirical literature. Oxford surveys in information technology, 4(1), 257-309.

[17] Rubinstein, D. (2007). Standish group report: There's less development chaos today. Software Development Times, 1.

[18] Rockart, J. F. (1982). The changing role of the information systems executive: a critical success factors perspective. Massachusetts Institute of Technology.

[19] Rowlinson, S. 1999 . "Selection criteria." Procurement systems: A guide to best practice, S. Rowlinson and P. McDermott, eds., E and F.N.

[20] Avolio, B. J., \& Gardner, W. L. (2005). Authentic leadership development: Getting to the root of positive forms of leadership. The Leadership Quarterly, 16(3), 315-338.

[21] Flowers, S. (1996). Software failure: management failure: amazing stories and cautionary tales. John Wiley \& Sons, Inc..

[22] Reel, J. S. (1999). Critical success factors in software projects. Software, IEEE, 16(3), 18-23. 